\title{
8. NUCLEAR SHELLS AND THE ORIGIN OF THE ELEMENTS
}

\author{
P. E. NEMIROVSKY
}

\section{INTRODUCTION}

The recent development of nuclear models allows us to define our knowledge of the effective one-particle potentials. This information enables us to make an extrapolation and find the neutron stability boundary of nuclei, which is of great interest for the theories of the origin of elements.

In this report an extreme model of a short-time scale neutron outburst is considered. The scale of the outburst is too short for $\beta$-decays to take place. All nuclei existing before the outburst will absorb neutrons and form new species, until the nucleus is formed, for which the neutron binding energy becomes zero.

The atomic weights at which neutron capture stops must be especially abundant in the Universe. This extreme model explains the large abundance of the isotope ${ }^{56} \mathrm{Fe}$.

\section{NUCLEAR POTENTIALS}

The one-particle model of nuclear interaction had recently a considerable success. The complex potential proposed by Weisskopf, Feshbach and Porter [r] for the explanation of the neutron nuclear cross-sections turned out to be closely connected with the interaction of the odd nucleon with the even-even core of the nucleus.

The introduction of the potential with a diffuse boundary, instead of the square well, and the addition of a spin-orbital interaction term improves the agreement with the cross-section data $[2,3,4]$. It was shown that the same potential with a vanishing imaginary part describes rather well the one-particle stationary states of nuclei [5].

The following dependence of the potential on $r$ was taken:

$$
V=-V_{0} \frac{\mathrm{I}+i \zeta}{\mathrm{I}+\exp (r-R) / a}
$$

where

$$
V_{0}=\frac{Z}{A} V_{1}+\frac{N}{A} V_{2}
$$

and the ratio $V_{1} / V_{2}$ has to be found by experiment.

The spin-orbit coupling term was written in the form:

$$
V_{1}=-\frac{x}{r} \frac{d v}{d r}(\vec{l} \overrightarrow{\sigma)}
$$

\section{II. STABILITY BOUNDARIES OF THE NUCLEI}

The above-mentioned results allow us to investigate the neutron-enriched nuclei. For these nuclei there exists a different order of shell filling, when neutron binding energy becomes small. For instance, the $I d_{5 / 2}$ level crosses the $2 s$ level, which is deeper for small binding energies. Similarly the $x f_{5 / 2}$ level crosses the $2 p_{1 / 2}$ and the $3 s$ level crosses $I g_{7 / 2}$ and $2 d_{5 / 2}$ and approaches $1 g_{9 / 2}$. Therefore the principal magic numbers are displaced and do not agree with magic numbers for stable nuclei.

One may suppose that magic numbers corresponding to the closed shells at small $E_{n l j}$ will be $N=8,16,20,40,52,82$, I26. The numbers 28 and 50 should not be magic, because of the vicinity of $I f_{7 / 2}$ and $2 p_{3 / 2}$ and $I g_{8 / 2}$ and $3 s$ levels.

The stability boundary of nuclei may be found. For this purpose it is necessary to determine at which neutron number for the given proton number the binding energy 


\section{JOINT DISCUSSION}

becomes zero. Only the maximum estimate of this number may be found, when only closed shell nuclei are considered. Indeed, the binding energy might become zero before the neutron shell is closed. This possibility can be investigated with minor success only.

Therefore only maximum estimates are considered in this paper. For light nuclei, an experimental correction is made by means of a decrease of the theoretical binding energies by $2 \mathrm{MeV}$. In Table I the maximum possible neutron number is given as a function of $Z$. The $N_{\max }$ given in Table I may, naturally, be altered. The experimental data are not sufficiently accurate for choosing $V_{1} / V_{2}=3$, or $V_{1} / V_{2}=4$. Nevertheless some results may be obtained. It is evident that $N_{\max }$ is of the order of $0.7 A$ over the whole periodic system. At neutron shells closing the minimum, proton number must have a jump. This jump is especially obvious at the closing of the principal nuclear shells with $N=20,40,82$, I26, I84. We give no data about nuclei with $82<N<126$ and $126<N<184$, because in this region the nuclei are essentially non-spheric, the shell structure is disturbed and cannot be restored at small binding energies.

Table I

\begin{tabular}{lrrrrrrrrr}
\hline$Z$ & 8 & 9 & 10 & 11 & 12 & 13 & 14 & 15 & 16 \\
$N_{\max }$ & 16 & 20 & 20 & 20 & 28 & 28 & 34 & 34 & 40 \\
\hline$Z$ & 18 & 20 & 21 & 22 & 24 & 26 & 28 & 30 & 32 \\
$N_{\max }$ & 40 & 50 & 52 & 52 & 58 & 62 & 70 & 70 & 82 \\
\hline$Z$ & 39 & 36 & 50 & 52 & 54 & 56 & 58 & 70 & 82 \\
$N_{\max }$ & 82 & 82 & 126 & 126 & 126 & 126 & 126 & 184 & 184 \\
\hline
\end{tabular}

\section{SOME CONSIDERATIONS ABOUT THE ORIGIN OF NUCLEAR SPECIES}

The closing of the shell with 40 neutrons for $I 6$ protons might have essential importance for the problem of the origin of elements. At this neutron and proton number the radioactive isotope ${ }^{56} \mathrm{~S}$ is formed, which decays into ${ }^{56} \mathrm{Fe}$.

Let us consider a neutron outburst, which takes place during a short time-interval, that is smaller than the $\beta$-mean lives of all nuclei formed. Then each nucleus will absorb neutrons until the species for which the neutron binding energy becomes zero is formed. Therefore light elements can transform into nuclei with medium atomic weight. At shell closing, when the neutron binding energy becomes zero, the $\beta$-decay starts and nuclei of corresponding atomic weight are accumulated. In some $\beta$-decays, delayed neutrons may be emitted. This will lead to the accumulation of some quantity of nuclei with smaller atomic weight. The closing of neutron shell at 34 neutrons is not proved, because of the presence of pairing energy, which will lead to the mixing of $2 p_{1 / 2}$ and $I f_{5 / 2}$ level (the latter has a larger pairing energy).

It is possible that starting from Si the nuclei with forty neutrons are formed. Nevertheless such considerations lead to the over-abundance of nuclei in the vicinity of ${ }^{56} \mathrm{Fe}$.

If the outburst is of a larger duration the estimation of $\beta$-half lives of nuclei with maximum neutron number is necessary. The $\beta$-decay energies for nuclei with $N=N_{\max }$ will be of the order of $I_{5}-\mathrm{I} 8 \mathrm{MeV}$. For estimating it must be supposed that the $\beta$-transition is allowed and that the daughter nucleus has several suitable levels for the $\beta$-decay. Then about ten branches of $\beta$-decay with the same $f t$ may be present.

Assuming $\log f t=3.5$ for allowed transitions and $f \sim E_{0}^{b}$ where $E_{0}$ is the maximum energy of the electron, we obtain $t=\mathrm{IO}^{-2} \mathrm{sec}$ at $E=\mathrm{I}_{5} \mathrm{MeV}$ for a single transition. Dividing with number of branches we obtain: $t=\mathrm{IO}^{-3} \mathrm{sec}$.

Probably in most cases the outburst duration is longer than the shortest half-lives. Therefore, the neutron absorbtion chain may be interrupted before the $N_{\max }$ is reached. In this case the special properties of $A=56$ are lost. However, also in this case, a maximum in the region $50<A<60$ may be expected. Indeed, the neutron capture in Ar and Ca may 


\section{NUCLEOGENESIS IN STARS}

lead to nuclei in this region of $A$ (for lighter nuclei the capture will lead to the formation of $\mathrm{Ca}$ ) and the following elements are too rare and therefore a sharp decrease can be obtained.

It may be noted that in nuclei with the neutron number larger than twenty, the lowlying $p$-levels are always observed. When the binding energy is large, such levels disappear at $N=50$. For small binding energies they must not be present in nuclei with $N>40$. Thus in the region $20<N<40$ there always exist states in which $S$-neutrons can make transitions with the emission of dipole radiation.

The probabilities of such transitions are varying proportionally to $E^{3}$.

If the capture cross-section for a thermal neutron is equal to 0.2 barns for $E=6.5 \mathrm{MeV}$ $\left({ }^{40} \mathrm{Ca}\right)$ then for $E=2 \mathrm{MeV}$ the capture cross-section must be of the order of $5 \times 10^{-27} \mathrm{~cm}^{2}$. At an energy equal to $25 \mathrm{KeV}\left(3 \times 10^{8}\right.$ degrees) this cross-section will be equal to $5 \times 10^{30} \mathrm{~cm}^{2}$ (according to the $\mathrm{I} / v$ law). Really the cross-sections are so small only at the latest stages; at larger binding energies they are in the range $10^{-28}<\sigma<5 \times 10^{-30} \mathrm{~cm}^{2}$. If the capture cross-section is $10^{-29} \mathrm{~cm}^{2}$, then at the neutron flux $\mathrm{IO}^{96} \mathrm{~cm}^{-2} \mathrm{sec}^{-1}$ (that is at densities $5 \times 10^{21} \mathrm{~cm}^{-3}$ and velocities $\left.2 \times 10^{8} \mathrm{~cm} / \mathrm{sec}\right),\left(I-e^{-10}\right)=100 \%$ of the original substance will be burnt in a second. Almost all the substance will be transformed into nuclear species with atomic weights IO-I5 units larger. We see that the transformation of light nuclei into medium weight nuclei is possible at very probable neutron fluxes (when the neutron density is equal only to $\mathrm{IO}^{-5}-\mathrm{IO}^{-7}$ of the density of matter in stars). This is a consequence of the presence of dipole transitions in nuclei with $20<A<40$.

\section{REFERENCES}

[I] Porter, Feshbach and Weisskopff. Phys. Rev. 96, 448, 1954.

[2] Nemirovsky, P. E. J.exp. theor. Phys. 30, 55I, 1956.

[3] Nemirovsky, P. E. J.exp. theor. Phys. 32, I 143, I957.

[4] Nemirovsky, P. E. and Ælagin, P. J. exp. theor. Phys. 32, I583, 1957.

[5] Nemirovsky, P. E. J.exp. theor. Phys. 33, 746, 1957.

\section{ON THE QUESTION OF LIGHT ELEMENTS FORMATION IN STELLAR ATMOSPHERES}

\section{B. A. TVERSKOY}

It is well known that $\mathrm{De}, \mathrm{Li}, \mathrm{Be}$ and $\mathrm{B}$ cannot exist in stellar interiors in amounts that are more than negligible.

Fowler and Burbidges [x] are of the opinion that $\mathrm{D}, \mathrm{Li}, \mathrm{Be}$ and $\mathrm{B}$ might be formed by disintegration of heavier nuclei by fast protons (with energy of the order of roo $\mathrm{MeV}$ ) accelerated by variable magnetic fields in stellar atmospheres. It is probable that this process causes the anomalously high concentration of $\mathrm{Li}, \mathrm{Be}$ and $\mathrm{B}$ in cosmic rays.

It is easy to show that in conditions of local thermodynamic equilibrium (i.e. Maxwellian velocity distribution) [2] an acceleration of a small part of nuclei is impossible.

It follows that electromagnetic acceleration requires a preliminary transfer of a part of nuclei across the region of thermal electron velocities. A possible mechanism for such a process is the passage of a shock wave towards more rarefied regions of the stellar atmospheres [3].

If those non-Maxwellian particles get into a region of variable electromagnetic fields, they are able to produce a steady acceleration. Continuously gaining energy, a part of them may cause the disintegration of heavier nuclei (e.g. carbon) with $\mathrm{Li}, \mathrm{Be}$ and $\mathrm{B}$ formation.

It follows that the mechanism proposed in [x] explains the anomalously high contents of $\mathrm{Li}, \mathrm{Be}$ and $\mathrm{B}$ in cosmic rays. However, on the other hand, this anomaly is not connected with the true abundance of these nuclei in the Universe. 